\title{
Performance demonstration of fiber and wireless fronthaul combination with remote powering
}

\author{
Z. TAYQ ${ }^{1,2}$, P. CHANCLOU ${ }^{1}$, T. DIALLO ${ }^{1,2}$, K. GRZYBOWSKI $^{1}$, F. SALIOU ${ }^{1}$, S. GOSSELIN ${ }^{1}$, \\ O. FOUCAULT ${ }^{1}$, C. AUPETIT ${ }^{2}$, L.BELLOT ${ }^{3}$, T.BOUKOUR $^{3}$, J.C. PLUMECOQ $^{3}$, J. SAYED $^{3}$ \\ ${ }^{I}$ Orange Labs Networks, 2 Avenue P.Marzin, 22300 Lannion, France, ${ }^{2}$ Laboratoire XLIM, 16 rue d'Atlantis, 87068 Limoges CEDEX, France \\ ${ }^{3}$ EBLINK, 3-5 rue Marcel Pagnol, 91800 Boussy Saint-Antoine, France \\ zakaria.tayq@orange.com
}

\begin{abstract}
An architecture combining optical fiber and wireless fronthaul is introduced. Tests have been performed on a LTE fronthaul link measuring the impact of this combination on EVM, jitter and frequency accuracy.

OCIS codes: (060.2330) Fiber optics communications; (060.2360) Fiber optics links and subsystems
\end{abstract}

\section{Introduction}

In order to satisfy the dramatic growth of mobile data access, some operators are considering Centralized or Cloud Radio Access Network (C-RAN) as the key of their future deployments. C-RAN consists in separating the Base Band Unit (BBU) that handles the signal processing in the base station (BS) from the Remote Radio Head (RRH). The latter contains the radiofrequency components at the antenna site and, at the other end, a central office centralizes the BBUs associated to several RRHs. This centralization creates a new network segment between BBUs and RRHs called mobile fronthaul based on Digital Radio over Fiber (D-RoF), e.g. according to Common Public Radio Interface (CPRI) [1]. The very low latency between the BBUs enables easier implementation of Coordinated Multi-Point (CoMP) and the compact form of RRHs provides footprint and renting cost reduction.

The privileged solutions to implement fronthaul are passive solutions based on Coarse Wavelength Division Multiplexing (CWDM) [2]. We consider in this paper technological solutions to upgrade CWDM. Indeed, the use of colored Small Form-factor Pluggable (SFPs) in the BBUs and RRHs makes wavelength management difficult for the mobile operator [2]. Also, operations administration and management (OAM) aspects have to be taken into account in parallel with transport performance in order to have key performance indicators of this network. Demarcation points [3] must be defined to separate the responsibilities between the mobile operator and the fiber provider in addition to the implementation of a monitoring solution that allows detecting fronthaul link degradations. Another major problem encountered in fronthaul deployment is the occasional complication in the fiber deployment, due to obstacles such as highways or rivers. A wireless fronthaul system is then welcome [4]. Another problem is the fact that a passive fronthaul transport still needs active RRHs with power supply at antenna site. In the context of a plurality of micro cells to extend radio capacity or coverage, the access to power source could be problematic.

In this paper, we experimentally investigate an architecture addressing the previously exposed problems; it combines optical fiber and wireless fronthaul with remote power supply. Different performance measurements are carried out. The performed experimental setup is described and the obtained results are discussed.

\section{Architecture under test: experimental setup}

The proposed architecture offers a mix of fiber and wireless fronthaul solutions. In fact, transponders are used in the demarcation points; this allows using Black and White (B\&W) SFPs in the BBUs and RRHs and colored SFPs in the line as shown in Figure 1 while conserving the integrity of the CPRI frame (no encapsulation) with very low latency (4 ns each). Also, transponders provide wavelength monitoring by generating alarms in case of fault in an optical channel. Different CPRI links are passively aggregated using CWDM multiplexers that offer up to 18 channels. The wireless section is achieved with a microwave link operating at $5.8 \mathrm{GHz}$ and supporting up to $3 \times 20 \mathrm{MHz}$ LTE carriers with low latency. These wireless devices (consuming less than $50 \mathrm{~W}$ ) are remotely powered thanks to hybrid energy/optical cables. Indeed, 400 VDC power is remotely provided from a central site through the hybrid energy/optical cable within an $8 \mathrm{~km}$ radius and $400 \mathrm{VDC}$ to $48 \mathrm{VDC}$ converters are used to power the wireless devices. Remote powering could also be used to power supply several RRHs from a BBU hotel if wireless is not used, with a limit of $3 \mathrm{~kW}$ for $3 \mathrm{~km}$ distance and $10 \mathrm{~mm}^{2}$ cable section. For instance, a site comprising 3 RRHs consuming $350 \mathrm{~W}$ each and a transponder consuming $30 \mathrm{~W}$ can be remotely powered with $4 \mathrm{~km}$ reach using $6 \mathrm{~mm}^{2}$ section cable. This solution will decrease energy expenses because less onsite energy components and electricity subscription contracts will be needed. 
Figure 1 shows the performed setup to test the proposed architecture. At the transmitter side, a LTE signal is generated by a signal generator then converted to CPRI using an IQ box. These two components emulate the BBU at first before using a commercial BBU to confirm the measured values. Standardized E-TM3.3 LTE test model is used with $20 \mathrm{MHz}$ carrier bandwidth. We test the CPRI at $2.457 \mathrm{~Gb} / \mathrm{s}$ in the downlink direction.

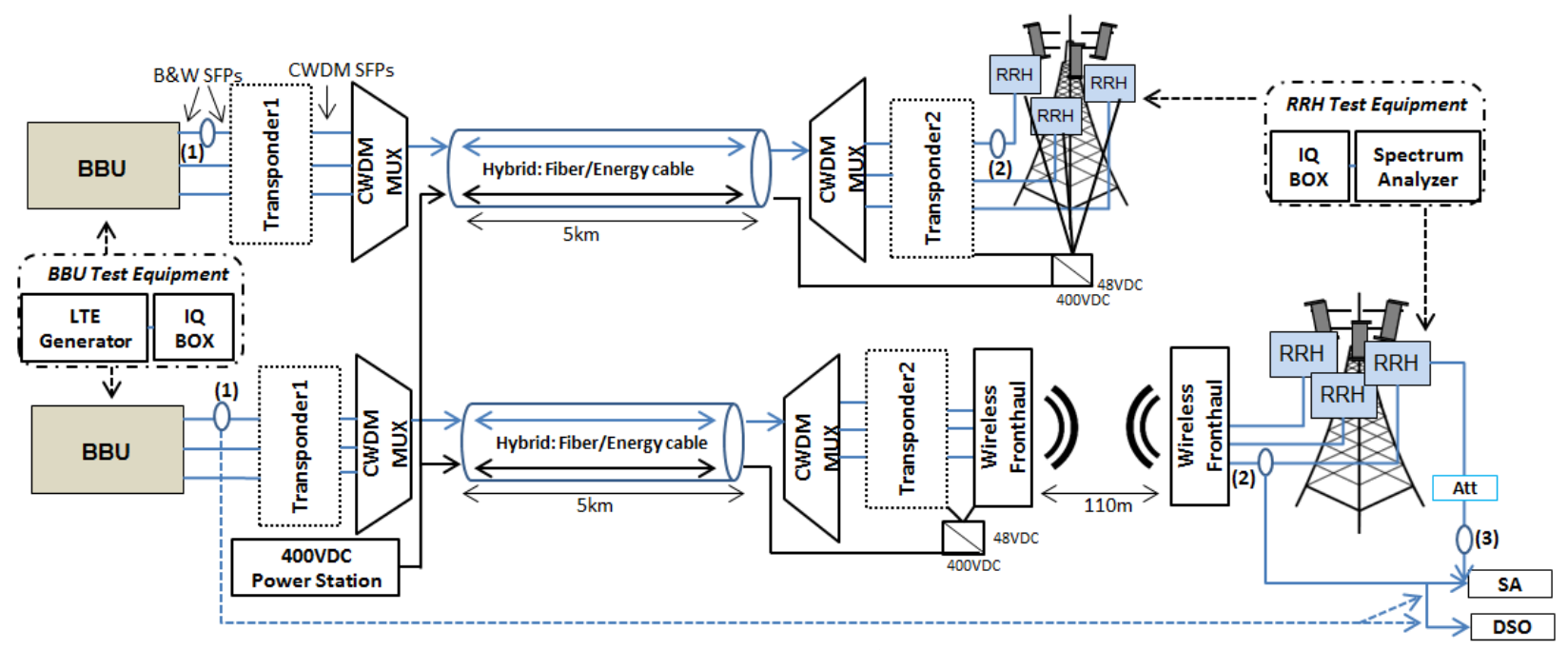

Figure 1: Experimental setup

Transponders followed by CWDM multiplexors are placed after the BBU transmitter and before the RRH. The fronthaul link includes $5 \mathrm{~km}$ of fiber and a wireless Over the Air (OTA) section of $110 \mathrm{~m}$, the Wireless Fronthaul Modules being set outdoor in Point-to-Point Line of Sight and remotely powered. The optical transmission is done with bidirectional single mode SFPs offering $23 \mathrm{~dB}$ of optical budget for a bit error rate of $10^{-12}$. At the receiver side, an IQ box is used to convert the CPRI to LTE before analysis with a Spectrum Analyzer (SA). After a photodiode, a Digital Storage Oscilloscope (DSO) allows measuring the jitter on the electric signal at the transmitter (1) and at the receiver (2) as shown in Figure 1. Finally, the SA permits to measure the frequency accuracy budget of the CPRI. Moreover, a frequency accuracy measurement of the LTE signal is performed with the SA at the output (3) of a commercial RRH operating in the $2.6 \mathrm{GHz}$ band.

\section{Experimental results}

We investigated the transmission performance from four aspects: error vector magnitude (EVM), jitter, CPRI frequency accuracy and LTE frequency accuracy.

First, we evaluated the EVM performance of the LTE signal. For QPSK, we measured $0.06 \%$ for optical fiber fronthaul without RRH, $1 \%$ with RRH only and $2.4 \%$ when combining fiber, wireless fronthaul and RRH. Hence an EVM contribution of $2.18 \%$ when adding a wireless section in the fronthaul link (value calculated on root mean square basis). The same result was obtained when using 64QAM modulation. This result is largely satisfactory since the maximum EVM value is $17.5 \%$ for QPSK and $9 \%$ for 64QAM according to 3GPP standard [5].

Secondly, we investigated the jitter introduced by equipment in the proposed architecture. As depicted in Table 1, some jitter is introduced in the link when combining wireless and optical fiber fronthaul without using transponders. In the case where transponders are inserted, we observe less jitter at the receiver due to resynchronization features of the transponder. In both cases, the measured jitter respects the CPRI specification as presented in Table 1.

Table 1: Jitter measurements for combined optical fiber and wireless fronthaul with and without transponders

\begin{tabular}{|c|c|c|c|}
\hline & $\begin{array}{c}\text { Measured values with } \\
\text { transponders }\end{array}$ & $\begin{array}{c}\text { Measured values without } \\
\text { transponders }\end{array}$ & $\begin{array}{c}\text { Maximum values according to the } \\
\text { CPRI specification }\end{array}$ \\
\hline Deterministic Jitter at Transmitter & 0.11 UI & 0.11 UI & 0.17 UI \\
\hline Total Jitter at Transmitter & 0.12 UI & 0.12 UI & 0.35 UI \\
\hline Deterministic Jitter at Receiver & 0.07 UI & 0.11 UI & 0.37 UI \\
\hline $\begin{array}{c}\text { Combined Deterministic and Random Jitter } \\
\text { at Receiver }\end{array}$ & 0.07 UI & 0.14 UI & 0.55 UI \\
\hline Total Jitter at Receiver & 0.07 UI & 0.14 UI & 0.65 UI \\
\hline
\end{tabular}


The introduced jitter could have an impact on the RRH synchronization [6]. Indeed, the central clock responsible for frequency generation in the RRH is synchronized with the clock of the CPRI signal. Therefore, inaccuracies in the transmitted clock could affect the precision of the generated LTE frequency thus the synchronization of User Equipment (UE). According to 3GPP, the most stringent requirement on LTE frequency accuracy on the air interface is $\pm 50 \mathrm{ppb}$ for wide area BS [5]. The maximum contribution of jitter from the CPRI to the base station frequency accuracy budget is limited to \pm 2 ppb conforming to the CPRI specification [1].

We started by investigating the CPRI frequency accuracy in the proposed architecture with and without wireless fronthaul in measurement points (1) and (2) as shown in Figure 1. The CPRI frequency deviation (FD) remains practically stable in the case of optical fiber fronthaul while we notice a FD difference of $0.4 \mathrm{ppb}$ between point (1) and point (2) when adding a wireless section. The measurement results are presented in Table 2; the measured FD at the reception $( \pm 1.13 \mathrm{ppb})$ respects the maximum value defined by the CPRI specification $( \pm 2 \mathrm{ppb})$.

Table 2: Frequency deviation at the output of the transmitter (BBU Tx) and at the input of the receiver (RRH Rx)

\begin{tabular}{|c|c|c|c|c|}
\hline \multicolumn{2}{|c|}{ Optical fiber fronthaul } & \multicolumn{2}{|c|}{ Combined optical fiber and wireless fronthaul } & $\begin{array}{c}\text { Maximum value according to } \\
\text { the CPRI specification }\end{array}$ \\
\hline BBU Tx & RRH Rx & BBU Tx & RRH Rx & $\pm 2 \mathrm{ppb}$ \\
\hline $0.73 \mathrm{ppb}$ & $\pm 0.78 \mathrm{ppb}$ & $\pm 0.73 \mathrm{ppb}$ & $\pm 1.13 \mathrm{ppb}$ & \pm \\
\hline
\end{tabular}

Finally, we measured LTE FD at the output of a commercial RRH with respect to nominal LTE frequency. As represented in figure 2 , we measure $\pm 3.8 \mathrm{ppb}$ FD with the proposed architecture while we obtain $\pm 1.4 \mathrm{ppb}$ when using dark fiber. The results respect the $3 \mathrm{GPP}$ standard that limits the maximum FD value of LTE at $\pm 50 \mathrm{ppb}$.
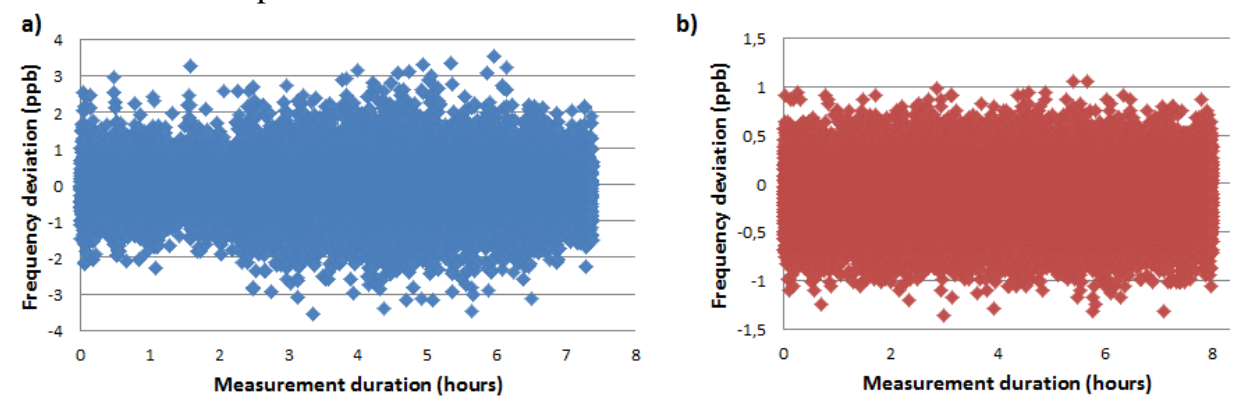

Figure 2: a) Frequency deviation measured on RRH radio output using fiber plus wireless fronthaul

b) Frequency deviation measured on RRH radio output for reference fronthaul (dark fiber)

Table 3 summarizes the performance measurements carried out with BBU and RRH test equipment considering the contribution of each network section to the measurements. These values were confirmed with field tests on commercial BBUs and RRHs. Latency is a dimensioning parameter that we need to consider as well. CoMP implementation for LTE-A requires a maximum round trip delay of $400 \mu$ s i.e. $40 \mathrm{~km}$ optical fiber. This value is limited to $38 \mathrm{~km}$ when using the proposed architecture and configuration with active WDM and wireless systems.

Table 3: recap chart of measured performances

\begin{tabular}{|c|c|c|c|c|c|}
\hline & EVM & Latency w/o fiber delay $(5 \mu \mathrm{s} / \mathrm{km})$ & Added jitter & CPRI FD & LTE FD \\
\hline Passive WDM: Fiber & $1 \%$ & 0 & 0 & $\pm 0.77 \mathrm{ppb}$ & $\pm 1.4 \mathrm{ppb}$ \\
\hline Active WDM: Fiber & $1 \%$ & $8 \mathrm{~ns}$ & 0 & $\pm 0.78 \mathrm{ppb}$ & in progress \\
\hline $\begin{array}{c}\text { Passive WDM: Fiber and } \\
\text { wireless combination }\end{array}$ & $2.4 \%$ & $9100 \mathrm{~ns}$ (including OTA section) & $20 \mathrm{mUI}$ & $\pm 1.15 \mathrm{ppb}$ & in progress \\
\hline $\begin{array}{c}\text { Active WDM: Fiber and } \\
\text { wireless combination }\end{array}$ & $2.4 \%$ & $9108 \mathrm{~ns}$ (including OTA section) & 0 & $\pm 1.13 \mathrm{ppb}$ & $\pm 3.8 \mathrm{ppb}$ \\
\hline
\end{tabular}

\section{Conclusion}

In this paper we proposed an architecture combining optical fiber and wireless fronthaul with a remote powering solution. End to end performance in terms of EVM, jitter and frequency accuracy was demonstrated in the lab and on a LTE commercial fronthaul. The obtained results are compliant with CPRI specification and 3GPP standard.

This work was supported by European projects COMBO, iCIRRUS and Xhaul. The authors wish to thank Infinera (TPMRHEX-L/16G) for their technical support.

[1] CPRI Interface Specification, v. 6.1, July $1^{\text {st }}, 2014$

[2] A.Pizzinat, P.Chanclou, F.Saliou, T.Diallo, "Things You Should Know About Fronthaul“", Journal of Lightwave Technologie, (2015)

[3] P.Chanclou,"Optical Fiber Solution for mobile Fronthaul to Achieve C-RAN," Proc.FuNeMS (2013)

[4] A.Rolland, RAN world, Fronthaul - Backhaul Working Group, 29-30 Sept. 2015, Cologne, Germany

[5] 3GPP TS 36.104: E-UTRA BS radio transmission and reception, section 6.5.1, Rel. 9, V9.8.0, June 2011.

[6] T. A. Diallo, A. Pizzinat, P. Chanclou, F. Saliou, F. Deletre, C. Aupetit-Berthelemot, "Jitter impact on mobile fronthaul links“"OFC (2014) 